\title{
Differential Diagnosis in Patients with HRCT Patterns Suspected for COVID-19 Pneumonia and RT-PCR Negative: A Multidisciplinary Approach for an Appropriate Management
}

\section{D'Onofrio Renato ( $\square$ renato.donofrio@studio.unibo.it)}

Department of Radiology, University of Bologna, Ospedale Sant'Orsola-Malpighi, Bologna, Italy

D'Andreta Michela

Department of Radiology, University of Bologna, Ospedale Sant'Orsola-Malpighi, Bologna, Italy

Modolon Cecilia

Department of Radiology, University of Bologna, Ospedale Sant'Orsola-Malpighi, Bologna, Italy

Rimondi Maria Rita

Department of Radiology, University of Bologna, Ospedale Sant'Orsola-Malpighi, Bologna, Italy

\section{Poggi Cristina}

Department of Radiology, University of Bologna, Ospedale Sant'Orsola-Malpighi, Bologna, Italy

\section{Di Scioscio Valerio}

Department of Radiology, University of Bologna, Ospedale Sant'Orsola-Malpighi, Bologna, Italy

\section{Trapani Filippo}

Infective Disease Unit, University of Bologna, Policlinico Sant'Orsola-Malpighi, Bologna, Italy

\section{Attard Luciano}

Infective Disease Unit, University of Bologna, Policlinico Sant'Orsola-Malpighi, Bologna, Italy

\section{Nava Stefano}

Respiratory and Critical Care Unit, University of Bologna, Policlinico Sant'Orsola-Malpighi, Bologna, Italy

\section{Monteduro Francesco}

Department of Radiology, University of Bologna, Ospedale Sant'Orsola-Malpighi, Bologna, Italy

\section{Case Report}

Keywords: COVID-19, RT-PCR, HRTC, GGO, multidisciplinary team

Posted Date: August 4th, 2020

DOI: https://doi.org/10.21203/rs.3.rs-51653/v1

License: (a) This work is licensed under a Creative Commons Attribution 4.0 International License. 
Page 2/14 


\section{Abstract}

Since 11th March 2020, the World Health Organization has declared that coronavirus disease 2019 (COVID-19) caused by SARS-CoV-2 is a pandemic and a global public health emergency.

One of the main problems related to the rapid spread of the pandemic was the low sensitivity of the swabs that were used, resulting in high false negative rates, and the inability of HRCT to distinguish COVID-19 pneumonia from other types of pneumonia, resulting in diagnostic delays and increased infections in the hospital units. To deal with this problem, a multidisciplinary team has been created at the Sant'Orsola-Malpighi University Hospital in Bologna, with the aim of integrating the data of each patient framed as a "COVID suspect" and to get to a correct diagnosis. We analysed some exemplary cases of patients with negative swab but highly suggestive clinical and radiological characteristics for COVID-19 that, thanks to this multidisciplinary team, were then framed as other forms of pneumonia and successfully treated.

\section{Key Points}

- Currently HRCT is not considered a screening survey for COVID-19

- The essential element to make diagnosis of COVID-19 at HRTC is the presence of GGO

- In patients with HRTC patterns suspected for COVID-19 and RT-PCR negative, a multidisciplinary team is useful to distinguish COVID-19 patients from non-COVIDs

\section{Introduction}

Since December 2019, in a few months, the coronavirus disease 2019 (COVID-19) or SARS-CoV-2 has caused a pandemic disease and the rapidity which it has propagated with is due to its high capacity to spread through the area in environments [1].

Italy was one of the countries most affected by the pandemic caused by the SARS CoV-2 virus with maximum spread of infections in March and April 2020. To avoid the spread of the virus, the government imposed the lock down from 12th March to 4th May 2020. These measures led to a concrete reduction of the infection curve. In the following period there was the gradual reopening of production activities and the hospital as well got back to normality. Since the contagion index was not zeroed and the virus continued to circulate, it was necessary to quickly establish a differential diagnosis between infected and non-infected patients, creating dedicated pathways.

Currently the diagnosis is obtained by the correlation between the respiratory symptoms and the positivity for COVID-19 genomic test, reverse transcriptase-polymerase chain reaction (RT-PCR) [2].

All patients entering the emergency department (ED) underwent nasal pharyngeal swab for SARS-CoV-2, whose result was available in 24 - 48 hours. So, the patients who needed hospitalization were transferred 
to dedicated departments called "the COVID-19 suspect unit" to wait for the result of genomic test. The patients were then transferred to a COVID-19 unit or a non-COVID-19 unit.

A close correlation between the onset of symptoms and the diagnostic effectiveness of RT-PCR has been demonstrated, with highest swab sensitivity being reached into 3 days after the onset of symptoms related to the viral infection [3].

However, the false negative rate high is due to the low sensitivity of the genomic test in use, which is about $60 \%$, so it is inevitable that there may be false negative patients that in the absence of a clear virologic diagnosis, could be hospitalized in common areas and represent an important source of infection. [4] There are many factors that can affect the false negativity rate of the test: the location of the sample, the inadequacy, the low viral load, also in relation to the time of sampling, technical issues during the processing of the sample in the laboratory [5].

Actually, high resolution computed tomography (HRCT) is not considered a screening survey of patients accessing ED with respiratory symptoms, as it involves radiation exposure, the risk of virus spreading to health care workers, medical costs, and long time required for disinfection.

HRCT is suggested in patients with moderate to severe symptoms to assess the severity of the damage and pulmonary dysfunction and for excluding a potential false-negative outcome from a negative RT-PCR test result [2].

HRCT has a high sensitivity in detecting lesions (sensitivity range that varies between $93 \%$ [6] and $97 \%$ [7]) but low specificity (53\% [6] and 25\% [7]).

The essential element to make diagnosis of COVID-19 pneumonia is the presence of ground-glass opacity (GGO) [5] which is an area of hazy increased lung opacity, usually extensive, within which margins of pulmonary vessels may be unrecognizable, with preservation of bronchial and vascular margins. It is caused by partial fluid filling of airspaces, interstitial thickening (due to fluid, cells, and/or fibrosis), partial collapse of alveoli, increased capillary blood volume, or a combination of those, the common factor being the partial displacement of air. It is less opaque than consolidation, in which bronchovascular margins are obscured [8].

GGO is a common finding of many other types of pneumonia, both infectious and non-infectious.

So, it became necessary to create a multidisciplinary team to discuss and make decisions on the management of those patients with GGO at the CT scan, at least two negative swabs, but a strong clinic or laboratory suspect for COVID-19.

In our study, we illustrate some exemplary cases of possible differential diagnoses of GGO detected at CT scan of patients with suspected COVID-19 and negative swab, in order to provide the clinician with possible scenarios to make the diagnostic process faster. 


\section{Discussion}

We analyzed the HRTCs of 138 patients $(64 \mathrm{~F} / 74 \mathrm{M})$ with an average age of 70 years old who underwent chest CT for suspected COVID-19 pneumonia at ED of Sant'Orsola-Malpighi University Hospital in Bologna, between May and June 2020. All patients underwent a medical examination, blood tests, serological test, nasal pharyngeal swab for SARS-CoV-2 and HRTC. The inclusion criteria were a positive respiratory symptomatology with a median incubation period of 4 days (interquartile range, 2 to 7), the presence of normal or high levels of C-Reactive Protein (CRP), high levels of Lactate Dehydrogenase (LDH) and Procalcitonin (PCT), lymphopenia with eosinopenia but no neutropenia and $\mathrm{PaO} 2 / \mathrm{FiO} 2$ ratio $(<300)$ [9][10], two or more negative swab tests and an indeterminate HRCT pattern. A multidisciplinary team was set up to analyze the various data and assess the risk of COVID-19 pneumonia infection for each patient who was transferred to a COVID-19 unit or a non-COVID-19 unit. The multidisciplinary team was composed by expert radiologists, infectiologists and pneumologists.

The main symptoms considered were typically respiratory (fever, cough, dyspnoea), but also gastrointestinal (nausea, diarrhoea), cardiological (syncope, heartbeat) and neurological (dysgeusia and anosmia).

The multidisciplinary team discussed the need for further diagnostic investigations, e.g. Filmarray, serological test and bronchoscopy with bronchoalveolar lavage (BAL).

This last one has demonstrated the highest rate of positive results for COVID-19, with sensitivity from 93 to $100 \%$ [11] [12]. Bronchoscopy should be performed in a negative pressure room to avoid aerosolisation of the virus, which would expose healthcare workers to a high risk of infection. In addition, bronchoscopy can lead to worsening respiratory failure, with the need to increase ventilatory support and therefore overload the use of resources. Moreover, it is not often possible to use because of the cardiopulmonary condition of the patient and the need of sedation or general anaesthesia in an intensive care unit. For these reasons one of the main purposes of the multidisciplinary team was to select patients who needed to perform BAL for a better diagnostic assessment.

The identification of an ongoing or past viral infection through the detection of antibodies is essential not only as an epidemiological tool for the study of seroprevalence but also as a complementary diagnostic test to those already present. It is based on the detection of IgM and IgG concentration and seroconversion time; the average day of seroconversion for both IgM and IgG it's 13 days after the onset of symptoms [13].

The review of HRCT images within the multidisciplinary team was carried by 10 and 20 years experienced cardiopulmonary radiologists. In this way it was possible to increase the diagnostic accuracy of the investigation.

By observing the HRCT characteristics and the data obtained, the multidisciplinary team took into consideration the possible differential diagnoses, allowing a correct management and therapy of all 
patients admitted to "suspected COVID" unit.

However, there is a wide variability of patterns which the disease can manifest with.

The Radiological Society of North America (RSNA) has defined four categories (typical, indeterminate, atypical, negative pattern) for reporting CT imaging findings potentially due to COVID-19, to create a standardized language [14].

HRCT does not allow a pathogen-specific diagnosis because there are many pathological pattern (e.g. other types of viral pneumonia), which have the same imaging characteristics of COVID-19 pneumonia [15]. As evidence of this, several studies have demonstrated that the diagnostic performance of chest CT scan shows a wide margin of variability, with high sensitivity (93\% [6] and 97\% [7]) but with low specificity (53\% [6] and 25\% [7]) and therefore it is an investigation burdened by the problem of differential diagnosis with other respiratory diseases. Moreover, about half of patients diagnosed with COVID-19 pneumonia may have normal HRTC in the first two days after onset of symptoms [16].

According to the Bayes theorem, since there is a high pre-test probability that cases of COVID-19 pneumonia will be detected during a pandemic, the post-test probability of the examination does increase.

However, this should not lead to underestimate the possible presence of other less common lung diseases, exposing patients to the risk of COVID-19 infection and leading to overloading COVID units.

The differential diagnosis of GGO is very wide, largely described in the literature. The study by Wallace et al. 2005 analyses the causes of isolated and diffuse GG (ID-GGO) and identifies 4 macro-categories with their statistically most relevant aetiologies: acute alveolar diseases (e.g. pulmonary oedema, acute respiratory distress syndrome, diffuse alveolar haemorrhage), opportunistic infections (e.g. Pneumocystis Carinii, Cytomegalovirus, Herpes Simplex Virus, Respiratory Syncytial Virus), chronic interstitial diseases (hypersensitivity pneumonia, desquamative interstitial pneumonia, respiratory bronchiolitis interstitial lung disease, nonspecific interstitial pneumonia, acute interstitial pneumonia, sarcoidosis) and other causes (drug toxicity, organizing pneumonia, neoplasia) [17]. In addition to the 4 categories, 5 clinical scenarios are described in which the ID-GGO is most often encountered: immunocompromised patients, patients undergoing bone marrow suppressing medications, patients with slowly progressive dyspnoea, patients with acutely developing dyspnoea and patient who are acutely ill.

More specifically, with regard to viral pneumonia, several studies show HRCT patterns strongly linked to the pathogenesis: micronodular pattern (e.g. chickenpox), pattern with centrolobular nodules and peripheral tree-in-bud (e.g. VRS), GGO pattern [18] [19] [20].

Upon macroscopic examination, the present literature indicates that the lungs of all patients with COVID19 pneumonia were heavy, congested, and oedematous, with patchy involvement. Histopathologic examination of lung biopsy tissues revealed GGO on HRCT matching with diffuse alveolar damage (recurrent findings were capillary congestion, interstitial and intra-alveolar oedema, dilated alveolar ducts 
and collapsed alveoli, hyaline membranes composed of serum proteins and condensed fibrin, and loss of pneumocytes) and therefore represents an early radiological sign of Acute Lung Injury [21] [22] [23].

Thanks to the integration of clinical laboratory data and the review of HRCT images by experienced radiologists, it was possible to frame each patient more accurately. In this way, all patients examined were found to be affected by diseases not related to COVID-19 infection, finally with $0 \%$ false positives.

\section{CASE REPORT}

Some study cases regarding some patients with RT-PCR negative test but "positive COVID-19 phenotype" who were examined in our institution are reported below.

Case N.1: 47-years-old female, anesthesiologist at COVID-19 unit of our Institute, went to the ED for an episode of hemoptysis during cough that forced her to bend forward, as referred by the patient herself. She had no fever, dyspnoea and no significant remote pathological history. Two negative COVID-19 swabs were performed, and HRCT showed small GGO in the middle lobe. There were small consolidations and mucus plugs in the middle lobar bronchus, without pleural effusion (Indeterminate pattern for COVID-19, based on RSNA guidelines, Fig. 1). A bronchoscopy revealed the presence of mucopurulent plugs. The patient was treated as a blood aspiration pneumonia. This is an atypical localization given by aspiration pneumonia that is usually located in the lower lobes [24]. In this case the GGO is in the middle lobar bronchus due to the forward bending of the patient who has deviated the blood coagulum into the middle lobar bronchus. The nature of the bleeding remains unknown.

Case N.2: 64-year-old male patient went to the ED for dry cough, dyspnoea and desaturation ( $84 \%$ in room air), without fever. In the past few months, he was hospitalized for bilateral pneumonia due to respiratory syncytial virus (RSV), found in the microbiological examination of BAL. Three COVID-19 swabs were performed, with negative results. HRCT showed extensive bilateral GGO with associated crazy-paving (Fig.2). Computed tomography findings could not rule out the possibility of COVID-19. He underwent BAL, resulted positive for RSV; therefore, he was treated with Ribavirin and Meropenem.

Case N.3: 51 -year-old female patient affected by AIDS went to ED suffering asthenia, dyspnoea and fever $\left(T \max 37.5^{\circ}\right.$ ) for a few days, with a finding of oxygen saturation $92 \%$ in room air. Two COVID-19 swabs were performed, with negative results. Blood tests showed severe lymphocytopenia (CD $4=35 / \mu L$ ), with normal inflammatory indices. HRCT showed widespread bilateral peri-hilar and peripheral GGO areas and associated patchy lung-base consolidations, without crazy-paving and pleural effusion (Fig.3). Bronchoscopy with BAL was performed with positive result for Pneumocystis Carinii.

Case N.4: 66-year-old female patient went to ED for dry cough, tachycardia, fever ( $T$ max $\left.37.2^{\circ}\right)$, with history of pulmonary hypertension and mitral insufficiency. Two COVID-19 swabs were performed, with negative results. HRCT showed bilateral pleural effusion with associated diffuse GGO, smooth interlobular septal thickening and cardiomegaly (Indeterminate pattern, Fig.4). The radiological findings 
were strongly suggestive for acute heart failure, confirmed by the specialist evaluations and echocardiogram.

Case N.5: 70-year-old male patient went to ED for dyspnoea from 15 days without fever and chest pain. In his medical history he had prostate cancer with bone metastases, currently on radiometabolic (last cycle 4 days before access) and cortisone therapy. He performed two negative swabs for COVID-19; hematochemical tests showed normal values of RCP and elevated values of LDH. HRCT showed multiple areas of GGO at the upper and middle fields with subpleural arrangement (Fig.5) were detected, which showed dimensional increase at subsequent HRCT follow-up. The CT showed the presence of an osteolytic area referable to metastasis in the right scapula, which has undergone recent radiotherapy treatment.

He also underwent pulmonary perfusional scintigraphy that showed signs of peripheral microembolism in the areas of GGO.

The radiological pattern appeared strongly suspect for COVID-19 pneumonia, unlike the clinicallaboratory data that, in relation to the anamnestic data, have oriented the diagnosis for radiation-induced pneumonia.

Case N.6: 84-year-old female patient, current smoker, went to ED for dyspnoea for 2 days without fever. In her medical history she had pulmonary hypertension, severe aortic stenosis and diffuse micronodular interstitiopathy.

She performed two swabs, negative for COVID-19. In known usual interstitial pneumonia (UIP) pulmonary fibrosis, HRCT showed diffuse interstitial thickening of inter and intra-lobular septa, mainly in the peripheral area with associated subpleural bronchiolectasis and diffuse GGO areas, especially in the central region. (Fig. 6). The radiological findings did not allow to distinguish between evolution of the fibrotic pathology and viral over-infection.

The clinical-laboratory data have oriented the diagnosis for exacerbation of the known chronic interstitiopathy.

\section{Conclusion}

\section{TO SUM UP}

The clinical cases reported in this article show that, in patients that were tested negative to the swab, CT images alone often do not allow us to make a differential diagnosis between COVID-19 pneumonia and other diseases with interstitial involvement. For this reason it was necessary to integrate clinical, laboratory, anamnestic and radiological data, with the creation of this multidisciplinary team mentioned above, which has allowed an optimal management of these patients, allowing a short stay in the "suspected COVID" unit, with the result of avoiding potentially dangerous contacts between negative and positive COVIDs, and the rapid setting up of an adequate and more effective pharmacological therapy. 
It is important that measures adopted by our Institute will be maintained and strengthened in the next future, in anticipation of a possible second wave of the epidemic, where the timeliness of affected patients recognition and their isolation will be decisive in order to avoid a second pandemic.

\section{Declarations}

\section{Compliance with ethical standards:}

\section{Guarantor:}

The scientific guarantor of this publication is D'Onofrio Renato.

\section{Conflict of interest:}

The authors of this manuscript declare no relationships with any companies, whose products or services may be related to the subject matter of the article.

\section{Funding:}

The authors state that this work has not received any funding.

\section{Statistics and biometry:}

No complex statistical methods were necessary for this paper.

\section{Informed consent:}

Written informed consent was obtained from all subjects (patients) in this study.

\section{Ethical approval:}

Institutional Review Board approval was not required because is a review design.

\section{Study subjects or cohorts overlap:}

None.

\section{Methodology:}

- retrospective

- observational

- performed at one institution

\section{Abbreviations}


coronavirus disease 2019: COVID-19; reverse transcriptase-polymerase chain reaction: RT-PCR; emergency department: ED; high resolution computed tomography HRCT; ground-glass opacity: GGO.

\section{References}

[1] Kai-Wang To K, Tak-Yin Tsang O, Leung WS et al (2020) Temporal profiles of viral load in posterior oropharyngeal saliva samples and serum antibody responses during infection by SARS-CoV-2: an observational cohort study. Lancet Infect Disease 20: 565-74

[2] Koo HJ, Choi SH, Sung H., Choe J, Do KH (2020) RadioGraphics Update: Radiographic and CT Features of Viral Pneumonia. RadioGraphics 40: E8-E15

[3] Kucirka LM, Lauer SA, Laeyendecker O, Boon D, Lessler J (2020) Variation in False-Negative Rate of Reverse Transcriptase Polymerase Chain Reaction-Based SARS-CoV-2 Tests by Time Since Exposure. Ann Intern Med:M20-1495

[4] Vaghi A, Calderazzo M, Carone M, Confalonieri M, Dal Farra F, Dottorini M, Gasparini S, Poletti V, Renda T, Scala R, Zuccatosta L (2020) AIPO - ITS - Approccio pragmatico alla diagnosi di polmonite da SARSCoV-2 [COVID-19] http://www.aiponet.it

[5]- Loeffelholz MJ, Tang YW (2020) Laboratory diagnosis of emerging human coronavirus infections the state of the art. Emerg Microbes Infect 2020; 9:747-56.

[6] Wen Z, Chi Y, Zhang L, et al (2020) Coronavirus disease 2019: initial detection on chest CT in a retrospective multicenter study of 103 Chinese subjects. Radiology Cardiothoracic Imaging. DOI:10.1148/ryct.2020200092

[7] Ai T, Yang Z, Hou H, et al (2020) Correlation of chest CT and RT-PCR testing in coronavirus disease 2019 (COVID-19) in China: a report of 1014 cases. Radiology:200642.

[8] Hansell DM, Bankier AA, MacMahon H, McLoud TC, Muller NL, Remy J (2008) Fleischner Society: Glossary of Terms for Thoracic Imaging. Radiology:246:697-722

[9] Guan W, Ni Z, Hu Y, Liang W, et al for the China Medical Treatment Expert Group for Covid-19 (2020) Clinical Characteristics of Coronavirus Disease 2019 in China. N Engl J Med; 382:1708-1720

[10] Lindsley AW, Schwartz JT, Rothenberg ME (2020) Eosinophil responses during COVID-19 infections and coronavirus vaccination. J Allergy Clin Immunol;146(1):1-7.

[11] Ost DA. (2020) Bronchoscopy in the Age of COVID-19. J Bronchology Interv Pulmonol DOI 10.1097/LBR.0000000000000682

[12] Wang W, Xu Y, Gao R, Lu R, Han K, Wu G, Tan W (2020) Detection of SARS-CoV-2 in Different Types of Clinical Specimens. JAMA;323(18):1843-1844 
[13] Long QX, Liu BZ, Deng HJ et al (2020) Antibody responses to SARS-CoV-2 in patients with COVID-19. Nat Med 26, 845-848.

[14] Simpson S, Kay FU , Abbara S, Bhalla S, Chung JH , Chung M , Henry TS, Kanne JP , Kligerman S, Ko JP, Litt H (2020) Radiological Society of North America Expert Consensus Statement on Reporting Chest CT Findings Related to COVID-19. Endorsed by the Society of Thoracic Radiology, the American College of Radiology, and RSNA. J Thorac Imaging DOI 10.1148/ryct.2020200152

[15] Dai WC, Zhang HW, Yu J, Xu HJ, Chen H, Luo SP, Zhang H, Liang LH, Wu XL, Lei Y, Lin F (2020) CT Imaging and Differential Diagnosis of COVID-19. Canadian Association of Radiologists Journal 71(2):195-200

[16] Bernheim A, Mei X, Huang M, Yang Y, Fayad ZA, Zhang N, Diao K, Lin B, Zhu X, Li K, Li S, Shan H, Jacobi A, Chung M (2020) Chest CT Findings in Coronavirus Disease 2019 (COVID-19): Relationship to Duration of Infection. Radiology 295:685-691

[17] Miller WT and Shah RM (2005) Isolated Diffuse Ground-Glass Opacity in Thoracic CT: Causes and Clinical Presentations. AJR; 184:613-622 0361-803X/05/1842-613

[18] Koo HJ, Lim S, Choe J, Choi SH, Sung H, Do KH (2018) Radiographic and CT Features of Viral Pneumonia. RadioGraphics; 38:719-739

[19] Ryu K, Takayanagi N, Ishiguro T, Kanauchi T, Kawate E, Kagiyama N, Sugita Y (2015) Etiology and Outcome of Diffuse Acute Infectious Bronchiolitis in Adults. Ann Am Thorac Soc Vol 12, No 12, pp 17811787

[20] Engeler CE, Tashjian JH, Trenkner SW, Walsh JW (1993) Ground-Glass Opacity of the Lung Parenchyma: A Guide to Analysis with High-Resolution CT. AJR; 160:249-251 0361-803X/93/1602-0249

[21] Carsana L, Sonzogni A, Nasr A, Rossi RS, Pellegrinelli A, Zerbi P, Rech R, Colombo R, Antinori S, Corbellino M, Galli M, Catena E, Tosoni A, Gianatti A, Nebuloni M (2020) Pulmonary post-mortem findings in a series of COVID-19 cases from northern Italy: a two-centre descriptive study. Lancet Infect Dis DOI:10.1101/2020.04.19.20054262

[22] Elicker BM; Jones KT; Naeger DM; Frank J (2016) Imaging of Acute Lung Injury. Radiol Clin North Am.; 54(6):1119-1132

[23] Zhang H, Zhou P, Hu M, et al (2020) Histopathologic changes and SARS-CoV-2 immunostaining in the lung of a patient with COVID-19. Ann Inter Med; 172: 629-32

[24] Hu X, Lee JS, Pianosi PT, Ryu JH (2015) Aspiration-Related Pulmonary Syndromes. CHEST; 147 (3): $815-823$. 


\section{Figures}

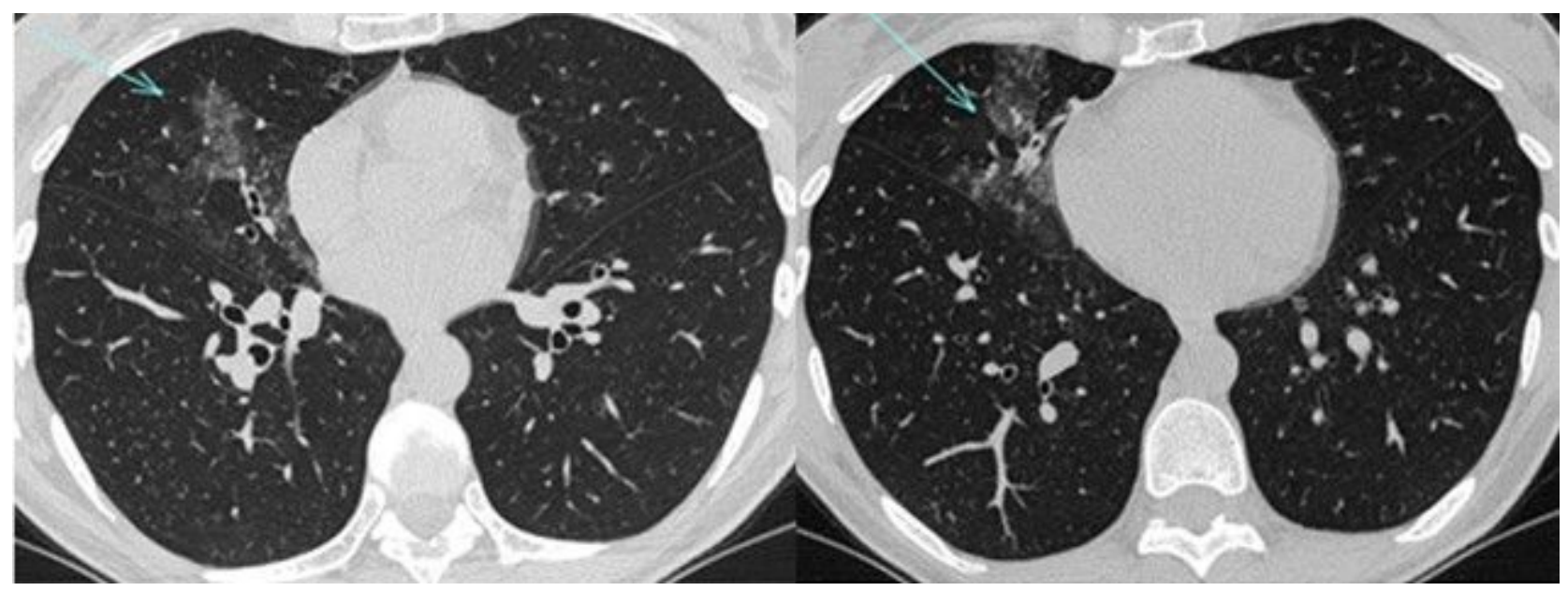

\section{Figure 1}

47-years-old female: HRCT showed small ground glass opacity (blue arrow) in the middle lobe with subpleural area sparing, small consolidation and mucus plugs in the middle lobar bronchus, without pleural effusion.

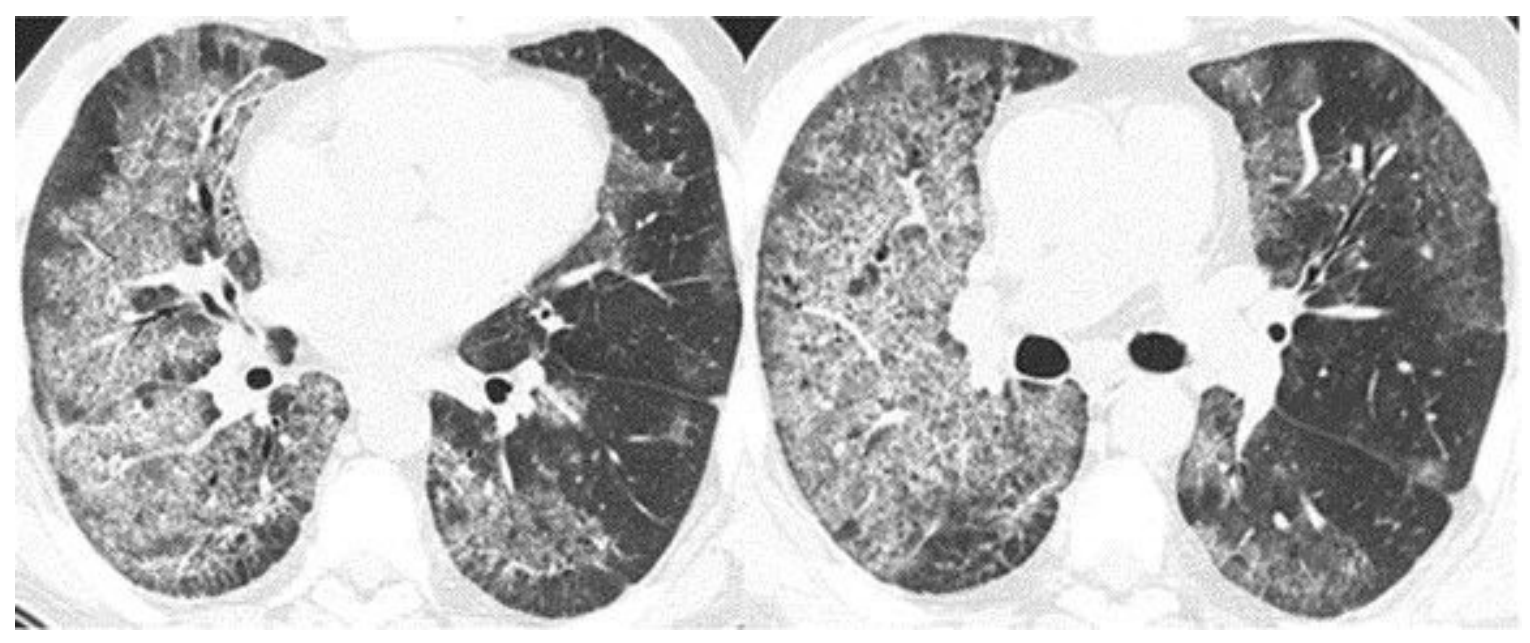

\section{Figure 2}

A 64-year-old male: HRCT showed extensive bilateral GGO with associated crazy-paving and consolidative basal lesions. 

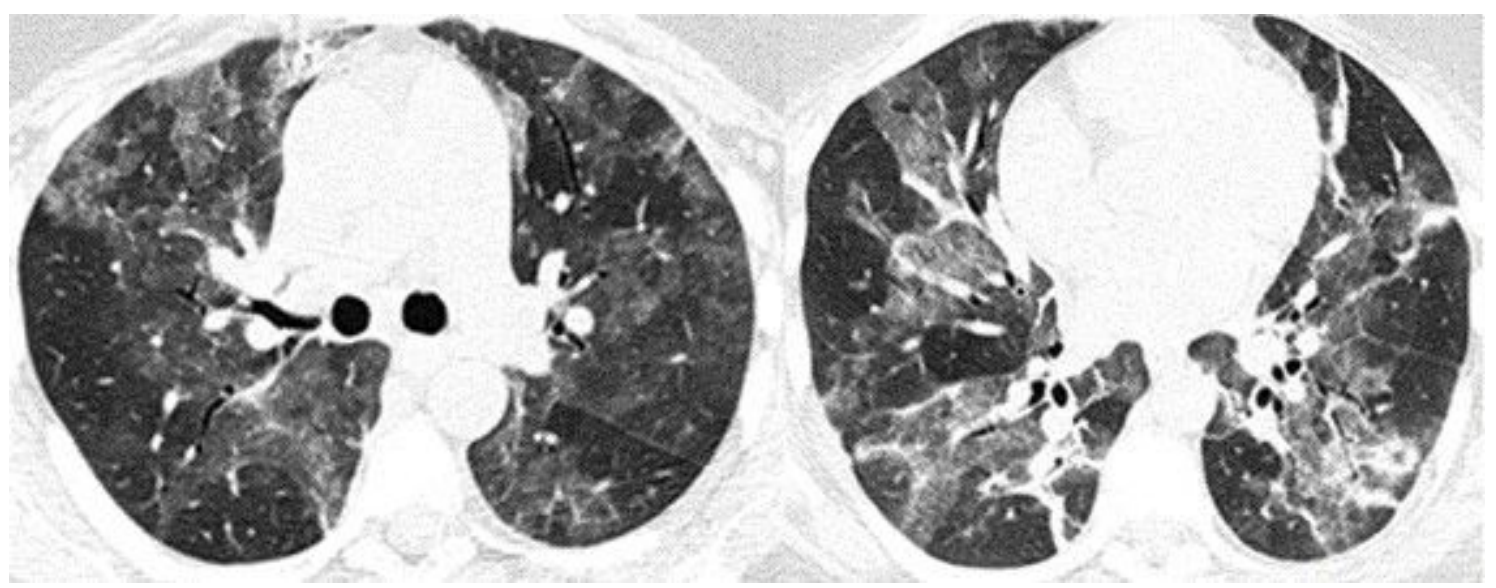

\section{Figure 3}

51-year-old female: HRCT showed widespread bilateral peri-hilar and peripheral GGO areas and associated patchy lung-base consolidations, without crazy-paving and pleural effusion.

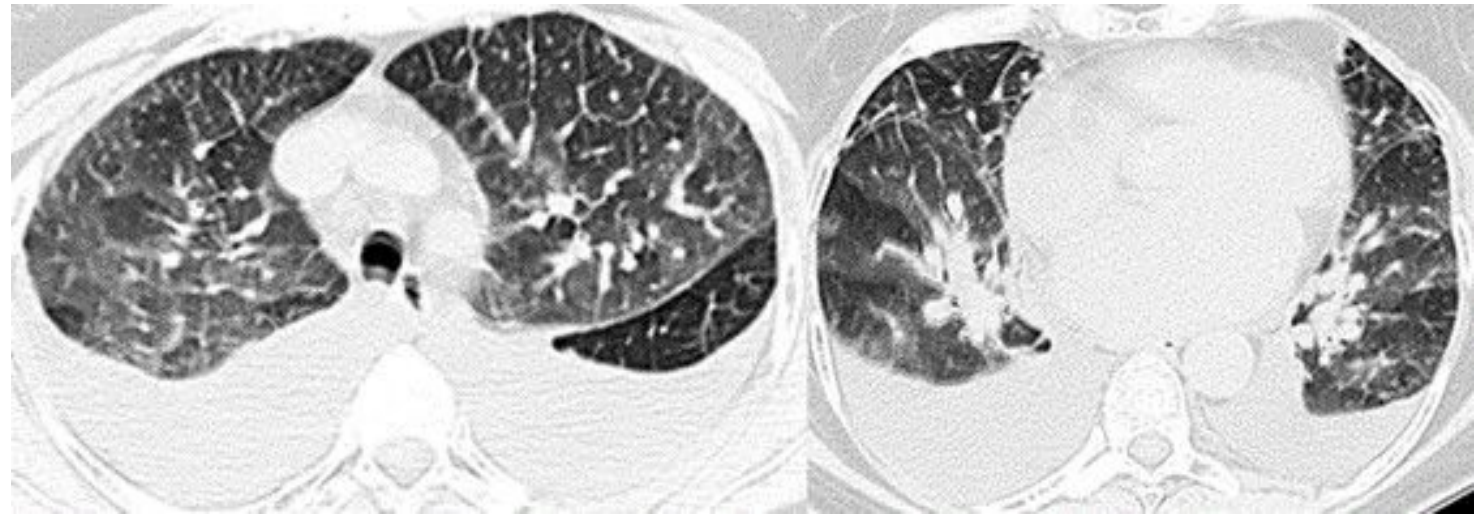

Figure 4

A 66-year-old female: HRCT showed bilateral pleural effusion with associated diffuse GGO, smooth interlobular septal thickening and cardiomegaly.
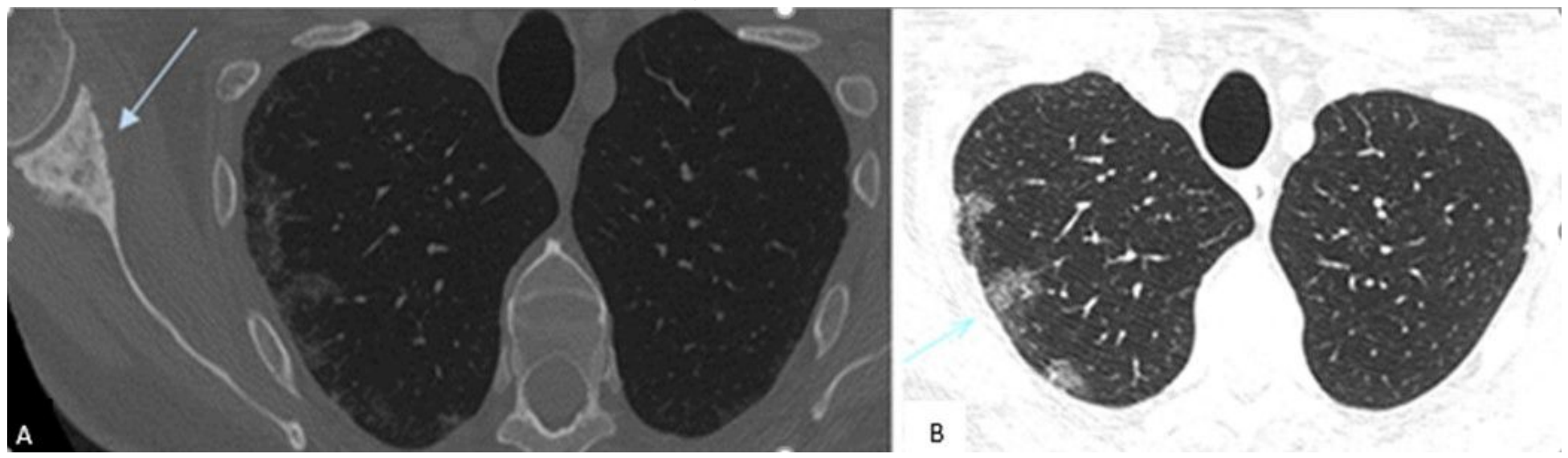

Figure 5 
A 70-year-old male: Fig 5A shows scapular metastasis from prostate cancer (thick arrow); Fig 5B shows multiple areas of GGO at the upper and middle fields with subpleural arrangement (thin arrow).

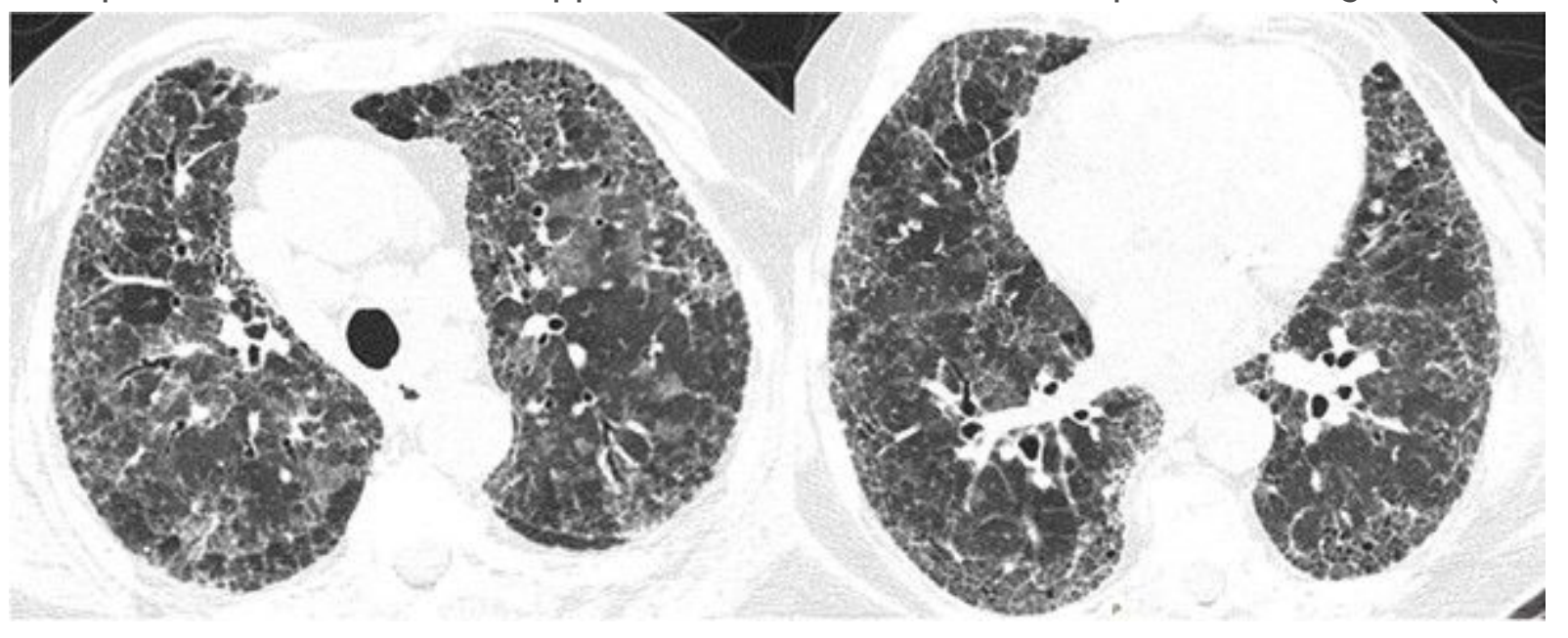

Figure 6

An 84-year-old female: HRCT showed thickening of interlobular and intralobular septa and central GGO areas. 